\title{
DETERMINACIÓN DE LAS CURVAS DE ESTADO ÚLTIMO DE AGOTAMIENTO EN SECCIONES RECTANGULARES DE HORMIGÓN ARMADO CON ARMADURA SIMÉTRICA O ASIMÉTRICA
}

\author{
(DETERMINATION OF ULTIMATE LIMIT STATE CURVES FOR RECTANGULAR \\ SECTIONS OF REINFORCED CONCRETE WITH SIMETRIC AND NON-SIMETRIC \\ REINFORCEMENT)
}

Sánchez Espinosa, E.; Garcimartín Molina, M. A., Dpto. de Construcción y Vias Rurales. UPM. López Domínguez, J. F., Dpto. de Matemática Aplicada a la Ingeniería Agronómica. UPM. E.T.S.I. AGRÓNOMOS, MADRID - ESPAÑA

\section{RESUMEN}

Se describe un programa de ordenador que permite la determinación de las curvas $M, N$ de agotamiento de secciones rectangulares de hormigón armado, con armadura simétrica o asimétrica, cualquiera que sea el tipo de hormigón y el recubrimiento mecánico de las armaduras para aceros normales. El programa, que se basa en el método de cálculo de la Parábola-Rectángulo, permite prescindir del empleo de ábacos teóricos y supone un procedimiento válido para la comprobación de estructuras de hormigón en condiciones reales de carga y armado.
SUMMARY

This paper shows an algorithm that allows to determine the $M, N$ curves at ultimate limit state for rectangular sections of reinforced concrete, with simetric and non-simetric reinforcement, for any kind of concrete, cover and steel reinforcement. The use of this program, based on the parabolic-rectangular model, makes innecessary to utilize of theoretical abacus and tables. It's a valid procedure to check concrete structures in real conditions of stress and reinforcement.

\section{FUNDAMENTOS E HIPÓTESIS PREVIAS}

El procedimiento usual de cálculo de secciones de hormigón armado es determinar las dimensiones de la sección y las áreas de las armaduras que proporcionan el equilibrio de fuerzas y de momentos, haciendo que la sección se encuentre en estado límite de agotamiento. Los valores de las áreas de las armaduras son en general teóricos, realizándose después un armado con un tamaño y número de redondos reales. Así se está en una curva real envolvente por encima de la teórica que, en principio, nos sitúa dentro de la seguridad.

Cuando se desea comprobar esa sección se calculan los axiles y momentos flectores últimos de reacción de la sección con armadura real y se comprueba que las solicitaciones a las que está sometida están por debajo de los axiles y momentos límites de agotamiento.
La determinación de los axiles y momentos de agotamiento de una sección se realiza en base a los materiales que se utilizan y a unas hipótesis previas de cálculo.

Se admite como diagrama de cálculo de tensión-deformación del acero el descrito en el art. 25.3 de la EH-88 y como diagrama de cálculo de tensión-deformación del hormigón el recogido en el art. 26.6 de la misma Norma Española (Fig. 2a).

Como hipótesis básicas de cálculo en agotamiento de secciones se adoptan las que se describen en el art. 36.1 de la EH-88.

El cálculo y la comprobación de secciones rectangulares de hormigón armado sometidas a solicitaciones normales se basan en las deformaciones límites de las 


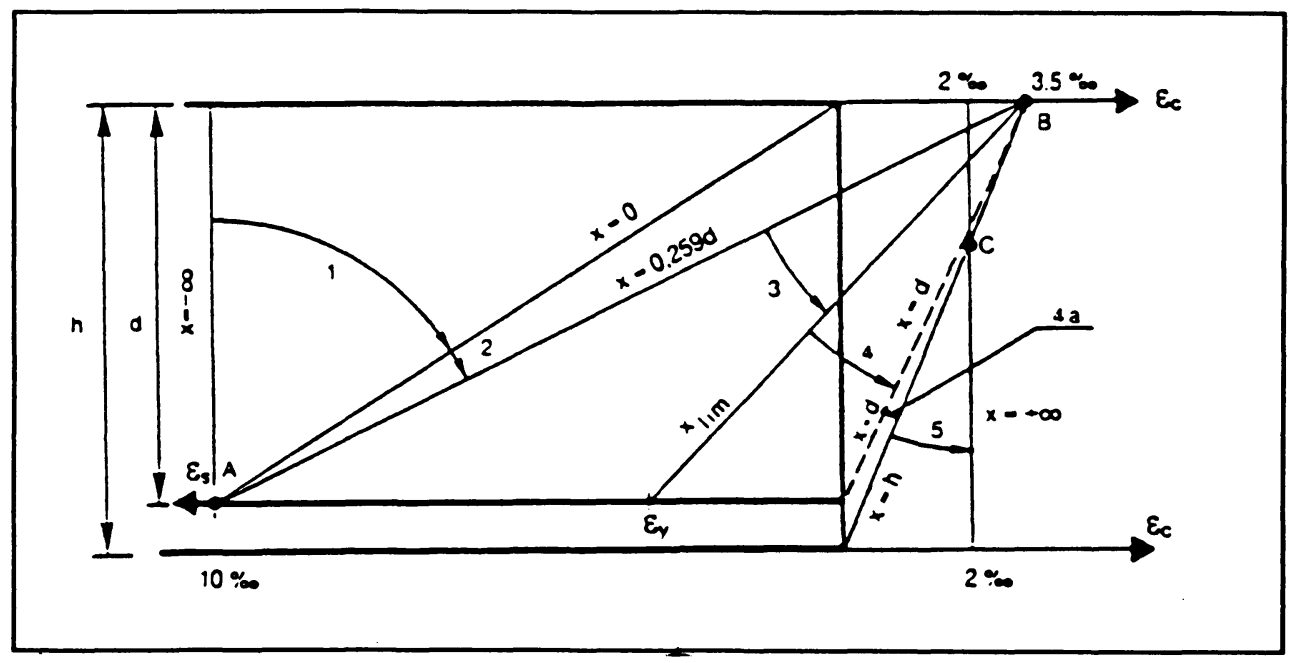

Fig. 1

secciones, que según la naturaleza de la solicitación, conducen a admitir los dominios del art. 36.2 EH-88.

Los dominios de deformación de la figura 1 se definen según las deformaciones del hormigón y el acero y la situación de la fibra neutra.

Los puntos $A$ y $B$ correspondientes a la máxima deformación del acero en tracción (10 por mil) y a la máxima deformación del hormigón (3,5 por mil) son de representación directa. El punto $\mathrm{C}$, sin embargo, se obtiene por proporcionalidad de triángulos, encontrándose a una distancia del borde superior de la sección de 3/7 de $h$ sobre la línea del 2 por mil, siendo h el canto total de la pieza.

Admitidas las caracteristicas de los materiales, las hipótesis previas de cálculo y los dominios de deformación se establecen las correspondientes ecuaciones de equilibrio y compatibilidad:

$$
\begin{array}{lc}
\Sigma_{F}=0 & N_{u}=\int_{0}^{b} b_{y} \cdot \sigma_{y} \cdot d y+A_{s 1} \cdot \sigma_{s 1}+A_{s 2} \cdot \sigma_{s 2} \\
\Sigma M=0 \quad N_{u} \cdot e_{1}=\int_{0}^{h} b_{y} \cdot \sigma_{y} \cdot(d-y) \cdot d y+A_{s 2} \cdot \sigma_{s 2} \cdot\left(d-d_{2}\right) \\
\frac{e_{c}}{x}=\frac{\varepsilon_{y}}{x-y}=\frac{\varepsilon_{s 1}}{x-d}=\frac{\varepsilon_{s 2}}{x-d_{2}}
\end{array}
$$

\section{PRISMA DE TENSIONES}

Para obtener la distribución de tensiones en una sección basta con conocer los valores de las deformaciones en sus bordes o la deformación en un borde y la profundidad de la fibra neutra. Así, haciendo una transformación afín del diagrama de cálculo parábolarectángulo teniendo como constante de afinidad la relación entre los dos puntos conocidos y las dimen- siones de la sección, se obtiene la distribución de tensiones y por tanto, todos los datos precisos para calcular su resultante y momento resultante, los cuales se corresponden con el volumen del prisma y su momento respecto al punto que interese (en general la armadura más traccionada).

La ecuación de la parábola de segundo grado del diagrama parábola-rectángulo es:

$$
\sigma_{c}=0.85 \cdot f_{c d}\left[1-\left(1+\frac{\epsilon_{c}}{0.002}\right)^{2}\right]
$$

Esta ecuación es válida para los valores de $\epsilon_{\mathrm{c}}$ por debajo del 2 por mil; para $\epsilon_{\mathrm{c}}$ entre el 2 por mil y el 3,5 por mil la tensión en el hormigón es constante e igual a $0,85 \cdot f_{c d}$. Es el tramo rectangular del diagrama adoptado.

El parámetro t de la figura 2 define la distancia desde el punto de tensión nula (posición de la fibra neutra), al punto correspondiente a una deformación del 2 por mil (en el que se tendría una tensión igual a $0,85 \cdot f_{c d}$ ).

Teniendo en cuenta el principio de Bernuilli sobre planitud de secciones, puede relacionarse $t$ con $x$ y con $\epsilon_{\mathrm{c}}$ de la siguiente forma:

$$
\frac{x}{-\epsilon_{c}}=\frac{t}{-0.002} \text { por tanto } \quad \frac{x}{t}=\frac{\epsilon_{c}}{-0.002}
$$

eliminando $\epsilon_{\mathrm{c}}$ entre (1) y (2) se obtiene la tensión del hormigón en función de la profundidad de la fibra neutra y del parámetro t:

$$
\sigma_{c}=0.85 \cdot f_{c d}\left[\frac{2 x}{t}-\frac{x^{2}}{t^{2}}\right]
$$




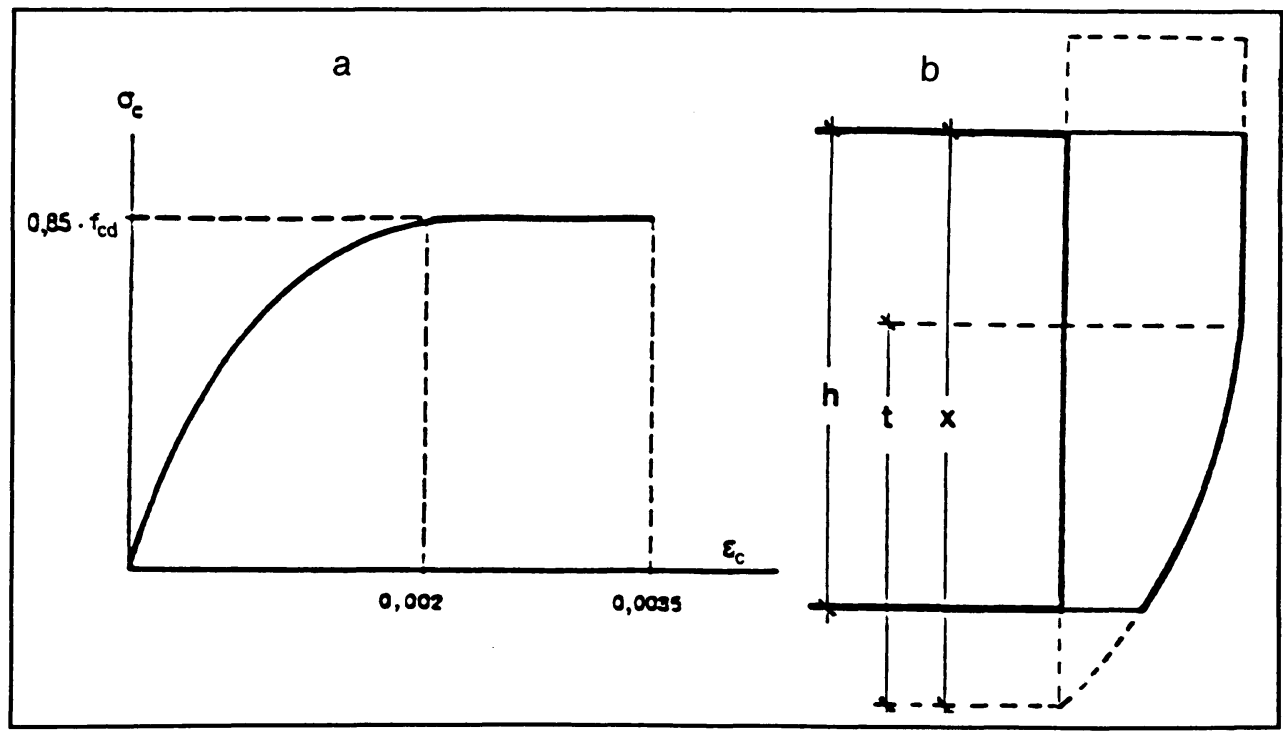

Fig. 2

Como el prisma es de altura constante b (anchura de la sección), el volumen es el resultado de multiplicar el área encerrada por la curva por dicha altura y el momento estático del primer orden el resultado de multiplicar el volumen por la distancia del centro de gravedad a la recta considerada.

Caso 1

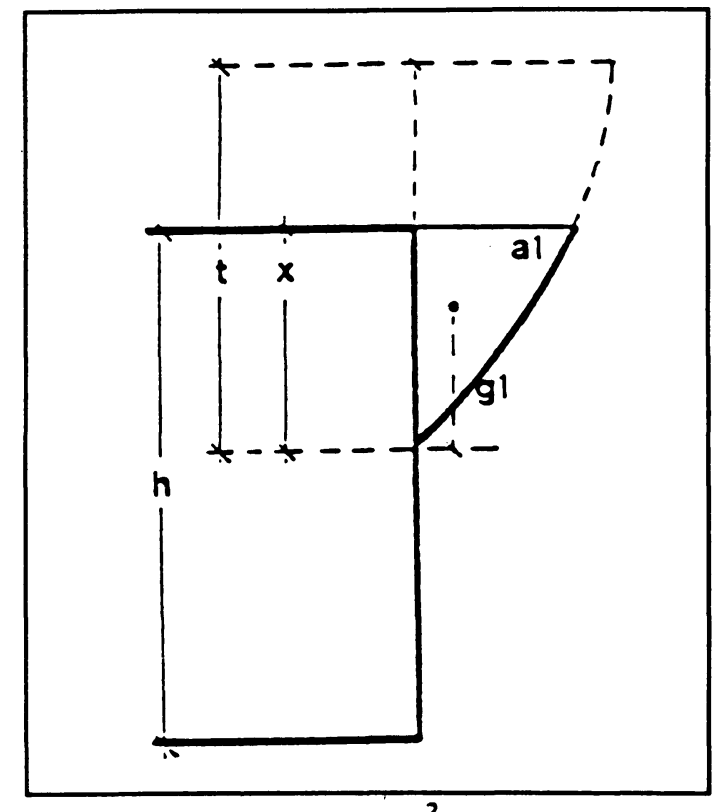

$a I=0.85 f_{c d} \frac{x^{2}}{t}\left(1-\frac{x}{3 t}\right)$

$$
g I=\frac{\left(\frac{2}{3}-\frac{x}{4 t}\right)}{\left(1-\frac{x}{3 t}\right)} x
$$

A continuación se presentan las áreas y los centros de gravedad para todos los casos en que se puede encontrar la posición de la fibra neutra:

Posición de la fibra neutra $0<x<h$ (Figuras 3 y 4 )

Caso 2

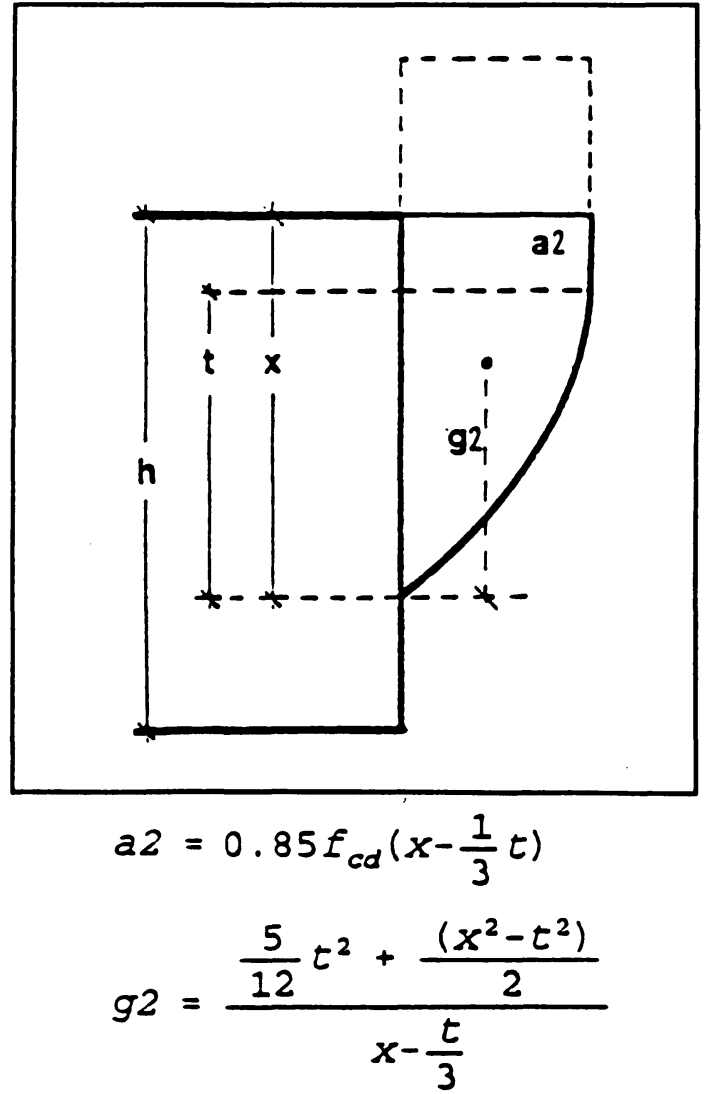

http://informesdelaconstruccion.revistas.csic.es 


\section{Caso 3}

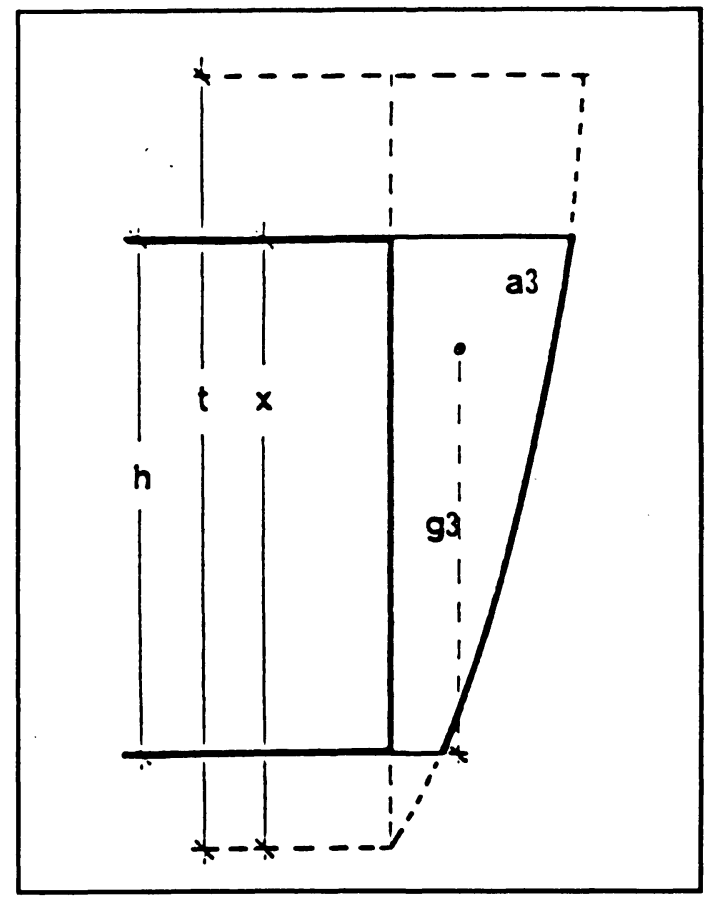

$a 3=0.85 \cdot f_{c d}\left[\frac{x^{2}}{t}\left(1-\frac{x}{3 t}\right)-\frac{(x-h)^{2}}{t}\left(1-\frac{x-h}{3 t}\right)\right]$ $g 3=\frac{x^{3}\left(\frac{2}{3}-\frac{x}{4 t}\right)-(x-h)^{3}\left(\frac{2}{3}-\frac{(x-h)}{4 t}\right)}{x^{2}\left(1-\frac{x}{3 t}\right)-(x-h)^{2}\left(1-\frac{(x-h)}{3 t}\right)}$

\section{Caso 4}



$$
\begin{gathered}
a 4=0.85 f_{c d}\left[\left(x-\frac{1}{3} t\right)-\frac{(x-h)^{2}}{t}\left(1-\frac{x-h}{3 t}\right)\right] \\
g 4=\frac{5 t^{4}+6 t^{2}\left(x^{2}-h^{2}\right)-8 t(x-h)^{3}+3(x-h)^{4}}{12 x t^{2}-4 t^{3}-12 t(x-h)^{2}+4(x-h)^{3}}
\end{gathered}
$$

Figs. 5 y 6

\section{SISTEMAS DE ECUACIONES}

Para la determinación de los valores de la solicitación resistente, (axil y momento flector resultante), en estados limites de agotamiento es necesario pivotar en los puntos A, B y $C$ antes descritos al hablar de los Dominios de la Deformación, obteniéndose los valores de las deformaciones del acero y del hormigón que sustituidos en el sistema de ecuaciones correspondiente permiten calcular los valores del axil y del momento de reacción de la sección. Se considera que una sección es válida cuando los valores de las solicitaciones son inferiores a los valores de reacción que puede proporcionar la sección.

Los sistemas de ecuaciones (equilibrio de fuerzas, equilibrio de momentos y condición de compatibilidad), antes mencionados, para las distintas situaciones que pueden presentarse son:

Pivote en $A\left(\epsilon_{1}=0,01\right)$

$0,01 \geq \epsilon_{2} \geq 0,01 \cdot\left(d^{\prime} / d\right)$

$$
\begin{gathered}
N-A_{s 1} \cdot \sigma_{s 1}-A_{s 2} \cdot \sigma_{s 2}=0 \\
N \cdot e_{1}-A_{s 2} \cdot \sigma_{s 2} \cdot\left(d-d^{\prime}\right)=0 \\
\frac{\epsilon_{1}}{x-d}=\frac{\epsilon_{2}}{x-d^{\prime}}
\end{gathered}
$$


$0,01 \cdot\left(d^{\prime} / d\right) \geq \epsilon_{2} \geq\left[0,012 \cdot\left(d^{\prime} / d\right)\right]-0,002$

$$
\begin{gathered}
N+a 1 \cdot b-A_{s 1} \cdot \sigma_{s 1}-A_{s 2} \cdot \sigma_{s 2}=0 \\
N \cdot e_{1}-a 1 \cdot b \cdot[g 1+(d-x)]-A_{s 2} \cdot \sigma_{s 2} \cdot\left(d-d^{\prime}\right)=0 \\
\frac{\epsilon_{1}}{x-d}=\frac{\epsilon_{2}}{x-d^{\prime}}=\frac{\epsilon_{c}}{x}=\frac{-0.002}{t}
\end{gathered}
$$

$\left[0,012 \cdot\left(d^{\prime} / d\right)\right] \cdot 0,002 \geq \epsilon_{2} \geq\left[0,0135 \cdot\left(d^{\prime} / d\right)\right] \cdot 0,0035$

$$
\begin{gathered}
N+a 2 \cdot b-A_{s 1} \cdot \sigma_{s 1}-A_{s 2} \cdot \sigma_{s 2}=0 \\
N \cdot e_{1}-a 2 \cdot b \cdot[g 2+(d-x)]-A_{s 2} \cdot \sigma_{s 2} \cdot\left(d-d^{\prime}\right)=0 \\
\frac{\epsilon_{1}}{x-d}=\frac{\epsilon_{2}}{x-d^{\prime}}=\frac{\epsilon_{c}}{x}=\frac{-0.002}{t}
\end{gathered}
$$

Pivote en $B\left(\epsilon_{\mathrm{c}}=0,0035\right)$

$\left[0,0135 \cdot\left(d^{\prime} / d\right)\right]-0,0035 \geq \epsilon_{2} \geq-(d / h) \cdot 0,0035$

$\epsilon_{1}=\left[\left(d / d^{\prime}\right) \cdot\left(0,0035+\epsilon_{2}\right)\right]-0,0035$

$$
\begin{gathered}
N+a 2 \cdot b-A_{s 1} \cdot \sigma_{s 1}-A_{s 2} \cdot \sigma_{s 2}=0 \\
N \cdot e_{1}-a 2 \cdot b \cdot[g 2+(d-x)]-A_{s 2} \cdot \sigma_{s 2} \cdot\left(d-d^{\prime}\right)=0 \\
\frac{\epsilon_{1}}{x-d}=\frac{\epsilon_{2}}{x-d^{\prime}}=\frac{\epsilon_{c}}{x}=\frac{-0.002}{t}
\end{gathered}
$$

\section{Pivote en C}

$-0,002 \geq \epsilon_{2} \geq-(\mathrm{d} / \mathrm{h}) \cdot 0,0035$

$\epsilon_{1}=\left[\frac{3 d^{\prime} \cdot 4 d}{3 d-4 d^{\prime}} \cdot\left(0,002+\epsilon_{1}\right)\right]-0,002$

$$
\begin{gathered}
N+a 3 \cdot b-A_{s 1} \cdot \sigma_{s 1}-A_{s 2} \cdot \sigma_{s 2}=0 \\
N \cdot e_{1}-a 4 \cdot b \cdot[g 4+(d-x)]-A_{s 2} \cdot \sigma_{s 2} \cdot\left(d-d^{\prime}\right)=0 \\
\frac{\epsilon_{1}}{x-d}=\frac{\epsilon_{2}}{x-d^{\prime}}=\frac{\epsilon_{c}}{x}=\frac{-0.002}{t}
\end{gathered}
$$

\section{PROGRAMA PIVOTABC DE ESTADOS LÍMITES ÚLTIMOS}

Una vez determinados los sistemas de ecuaciones para cada uno de los puntos de pivote se ha procedido a diseñar un programa que obtiene pares de valores N,M de la curva de Estados Límites de Agotamiento.
Los datos necesarios de la sección son los siguientes:

- Anchura b.

- Recubrimiento mecánico d'.

- Canto útil d.

- Canto total h, obtenido como suma de d y de d'.

- Módulo de elasticidad del acero E.

- Resistencia de cálculo del acero $f_{\text {yd }}$.

- Resistencia de cálculo del hormigón $\mathbf{f}_{\mathrm{cd}}$.

- Área de la armadura mas traccionada $\mathbf{A}_{\mathrm{s} \text {. }}$.

- Área de la armadura menos traccionada $\mathbf{A}_{\mathbf{s 2}}$.

Así, se pueden distinguir tres zonas de pares de valores $\left(\epsilon_{1}, \epsilon_{2}\right)$ dentro del programa.

La primera, de pivote en $\mathbf{A}$, incluye los pares de valores formados por $\epsilon_{1}=0,01$ y $\epsilon_{2}$ variando entre un valor que hace que la máxima deformación del hormigón sea $\epsilon_{c}=-0,0035$ (punto B) y un valor que es:

a) $\epsilon_{y}$ si la fibra neutra se encuentra en el intervalo $-\infty<x<0$.

b) El valor $\epsilon_{2}$ (mayor que $\epsilon_{y}$ ) tal que la fibra neutra se encuentre en el borde superior de la sección $(x=0)$.

Para valores de $\epsilon_{2}$ mayores que este límite nos encontramos en situación de rótula plástica, sin que existan variaciones en los valores de axil y momento flector, por lo que esta zona está representada por un punto único.

La segunda es de pivote en B siendo $\epsilon_{c}$ constante $e$ igual al 3,5 por mil. En tal situación, se considera un intervalo fijo para $\epsilon_{1}$ entre el punto $A\left(\epsilon_{1}=0,01\right)$ y el que hace que la recta de deformación pase por el punto $C\left(\epsilon_{1}=-\left(d^{\prime} \cdot 0,0035\right) / h\right)$. Con cada valor de $\epsilon_{1}$ de este intervalo se calcula el $\epsilon_{2}$ correspondiente para que la recta de deformación pase por $B$.

Por último, la tercera zona se compone de los pares de valores que hacen que la recta de deformación pase por el punto de pivote $\mathbf{C}$, para lo cual se hace variar $\epsilon_{1}$ entre - 0,002 y - (d'.0,0035)/h, calculando en cada caso el $\epsilon_{2}$ correspondiente.

Mediante un proceso iterativo se establece el par $\left(\epsilon_{1}, \epsilon_{2}\right)$ y se procede a determinar la profundidad de la fibra neutra, la deformación $\epsilon_{\mathrm{c}}$ del hormigón y el valor del parámetro t. En función de los valores que adop$\tan \epsilon_{\mathrm{c}} \mathrm{y} \times$ se selecciona el sistema de ecuaciones idóneo, con el que se obtienen los valores de axil y momento de cálculo $\left(\mathrm{N}, \mathrm{M}_{\mathrm{d}}\right)$.

Con objeto de obtener el valor del momento flector $M$, es necesario transformar $M_{d}$ mediante la ecuación:

$$
M(x)=N \cdot\left(\frac{M_{d}}{N}+\frac{d-d^{\prime}}{2}\right)
$$


El lenguaje utilizado para la aplicación informática ha sido QuickBASIC 4.5, para mayor claridad se incluyen los ordinogramas básicos del programa.

\section{APLICACIÓN}

Para mostrar el funcionamiento de este programa se ha realizado una aplicación para dos secciones de las mismas dimensiones con diferente armado. Las características de las secciones son:

- Canto total, $\mathbf{h}=30 \mathrm{~cm}$.

- Canto últil, $\mathbf{d}=26,6 \mathrm{~cm}$.

- Recubrimiento mecánico, d' $=3,4 \mathrm{~cm}$.

- Anchura de la sección, $\mathbf{b}=20 \mathrm{~cm}$.

- Hormigón H-200. Coeficiente de seguridad 1,5.

- Acero AEH -400 con $\mathrm{f}_{\mathrm{yk}}=4200 \mathrm{kp} / \mathrm{cm}^{2}$ y coeficiente de seguridad 1,15.

Sección a) Armado asimétrico:

$A_{\mathrm{s} 1}: 4$ redondos de $16 \mathrm{~mm}$.

$A_{\mathrm{s} 2}: 2$ redondos de $16 \mathrm{~mm}$.

Sección b) Armado simétrico:

$A_{\mathrm{s} 1}: 4$ redondos de $16 \mathrm{~mm}$.

$A_{\mathrm{s} 2}: 4$ redondos de $16 \mathrm{~mm}$.

Los pares axil-momento flector de agotamiento $(N, M)$ obtenidos ejecutando el programa, se almacenan en un fichero ASCII de forma que sea posible su captura por un programa de gráficos.

Empleando el programa Havard Graphics se ha realizado la representación de las curvas de Estado Límite de Agotamiento para las secciones antes descritas.

\section{CONCLUSIONES}

- El algoritmo propuesto presenta la ventaja de adaptarse a las hipótesis generales de comprobación de secciones y por tanto representa una solución ajustada al sentido físico del problema.

- El procedimiento propuesto constituye una solución efectiva para la comprobación de secciones con ordenador.

- La aplicación PIVOTABC permite la obtención de curvas de estado de agotamiento para secciones rectangulares con cualquier tipo de armadura simétrica o asimétrica y recubrimientos mecánicos variables.

\section{BiBLIOGRAFÍA}

ARIBERT, J. M.; WATTECAMPS, C. (1979). "Methode pratique commune de calcul elastique et aux etats limites ultimes des sections de Beton Arme en Flexion Simple, Composee et Deviee". Ann Inst Tech Batim Trav Publics n. ${ }^{\circ} 374, \mathrm{p}$ 93-130.

BRAGA, F.; DOLCE, M. (1980). "Grafici per la progettazione optimale di sezioni rettangolari in C.A. sollecitate allo stato limite ultimo da sforzo normale e momento flettente". Ind Ital Cem v 50 n. ${ }^{0}$ 7-8, p 511-520.

CAPRA, A. HAUTCOEUR, M. (1979). "Calcul en flexion simple ou composee a l'etat limite ultime des sections rectangulires en Beton Arme". Ann Inst Tech Batim Trav Publics $n .^{\circ} 375, \mathrm{p} 41-66$.

GARCIA MESEGUER, A. (1971). "Formules simplifiées". CEB, Bulletin d'Information n. ${ }^{\circ} 75$. Paris.

GARCIA MESEGUER, A. (1988). "Hormigón Armado. Cálculo en estados límites". U.N.E.D. Madrid.

JIMENEZ MONTOYA, P. (1972). "Universal tables for Designing rectangular sections in simple or composite Bending". CEB, Bulletin d'Information n. ${ }^{\circ} 82$. Paris.

JIMENEZ MONTOYA, P. MORAN, F. (1972). "Aproximation formulas". CEB, Bulletin d'Information n. 82 . Paris.

JIMENEZ MONTOYA, P. GARCIA MESEGUER, A. MORAN, F. (1987). "Hormigón Armado, 12ª . Edición". Gustavo Gili. Barcelona.

KOTSOVOS, M. D. (1988). "Compressive force path concept: basis for reinforced concrete ultimate limit state design". ACI Struct J v 85, n. ${ }^{\circ}$ 1, p. 68-75.

MORAN CABRE, F. (1972). "Cálculo de secciones de hormigón armado, sometidas a solicitaciones normales, en el estado límite último". Instituto Eduardo To. rroja. Madrid.

PAEZ BALACA, A. (1986). "Hormigón armado". Ed. Reverté. Barcelona.

\section{NORMAS Y REGLAMENTOS}

Comité Euro-Internacional del hormigón CEB-FIP. "Manual Bending and compression". 1972.

Comité Euro-Internacional del hormigón CEB. "Códigomodelo CEB-FIP para las estructuras de hormigón". 1978.

Commission of the European Communities. "Eurocode n. ${ }^{\circ}$ 2. Design of Concrete Structures". 1989.

MOPU. "Instrucción para el proyecto y la ejecución de obras de hormigón en masa o armado. EH-88". 1988. 
PIVOTE EN A, B Y C

Axiles y Momentos flectores

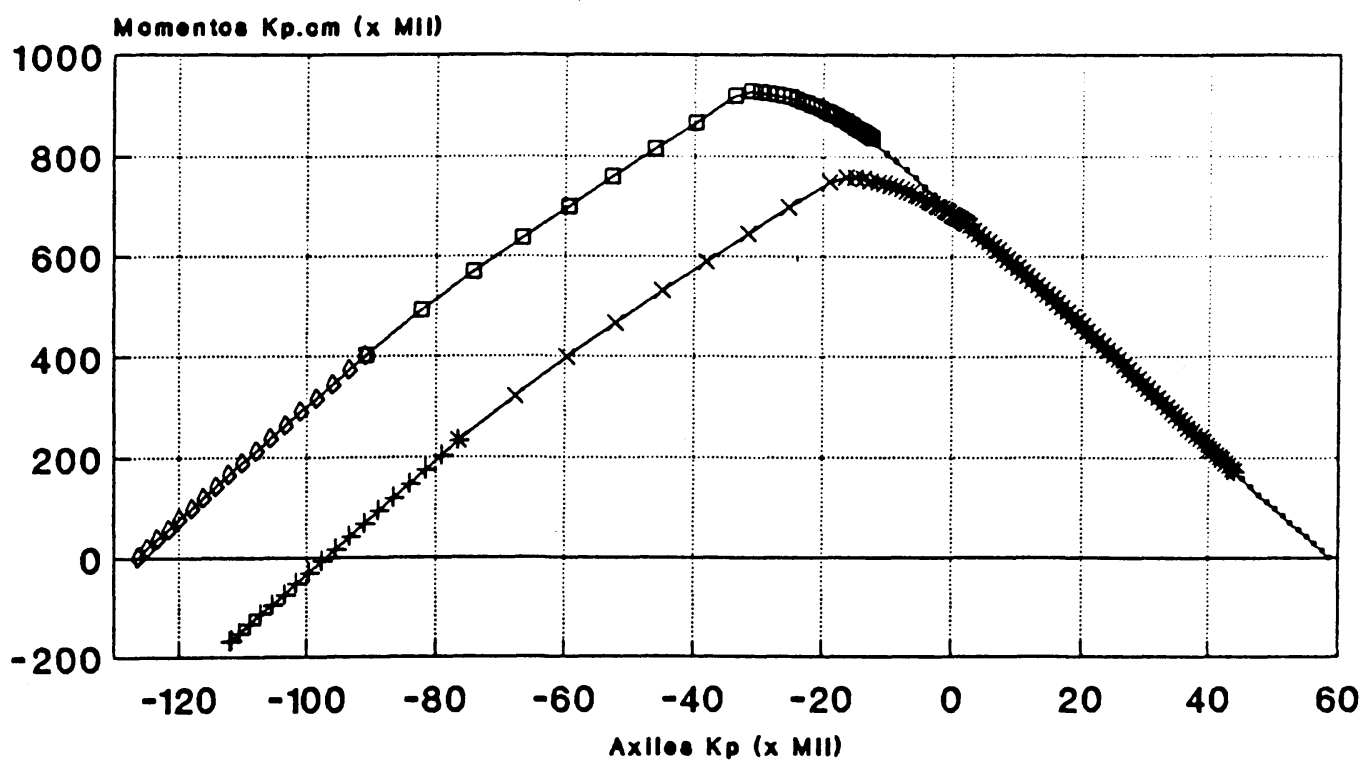

* Pto. A, A. no S. $\quad$ Pto. B, A. no S. $\quad$ Pto. C, A. no S.

$\longrightarrow$ Pto. A. A.S. $\rightarrow$ Pto. B. A.S. Pto. C, A.S.

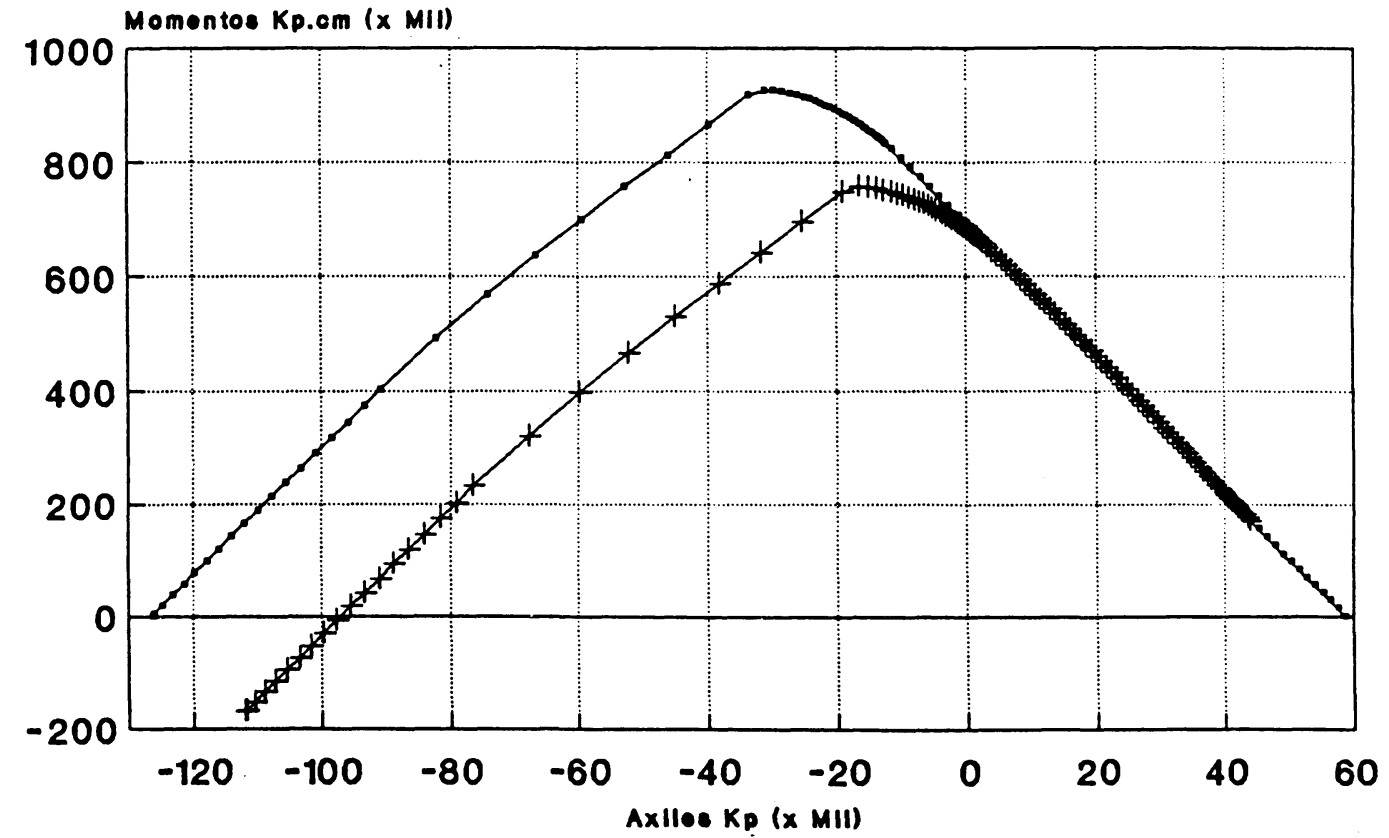


PROGRAMA PIVOTABC

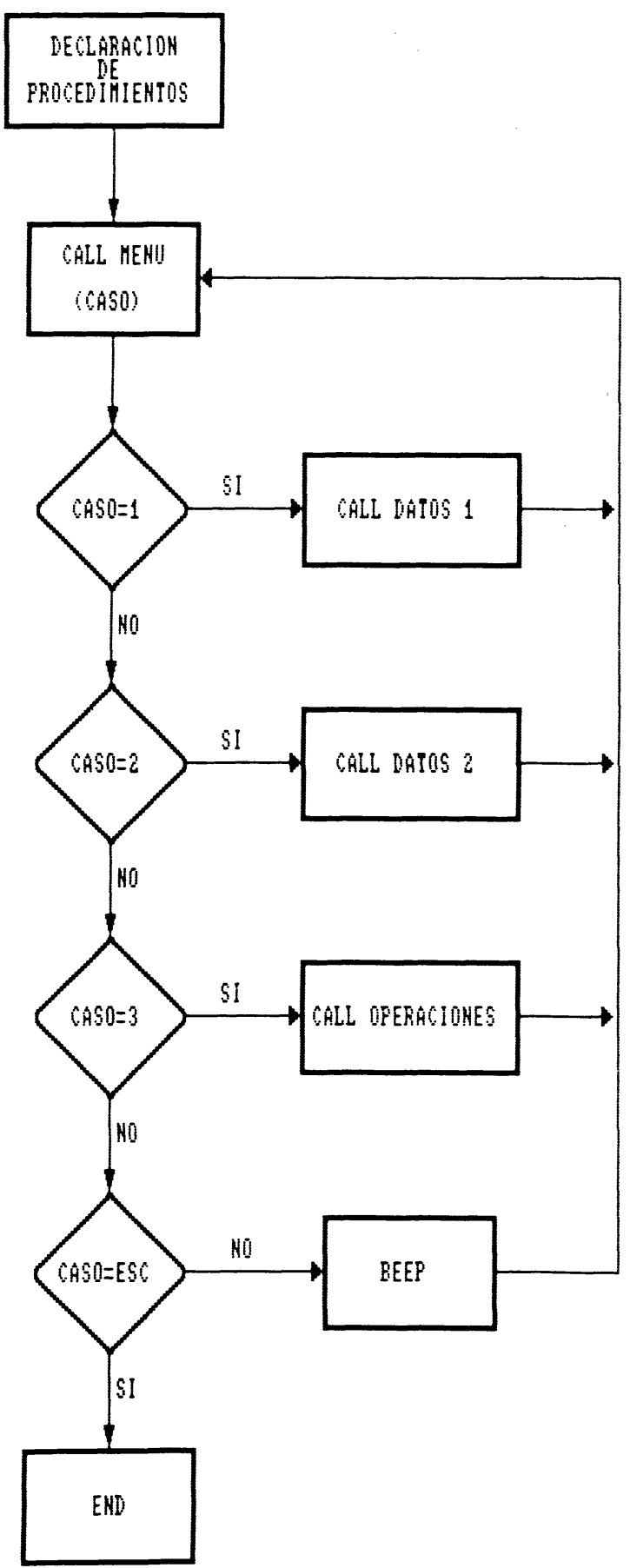

DATOS 1 Y DATOS 2

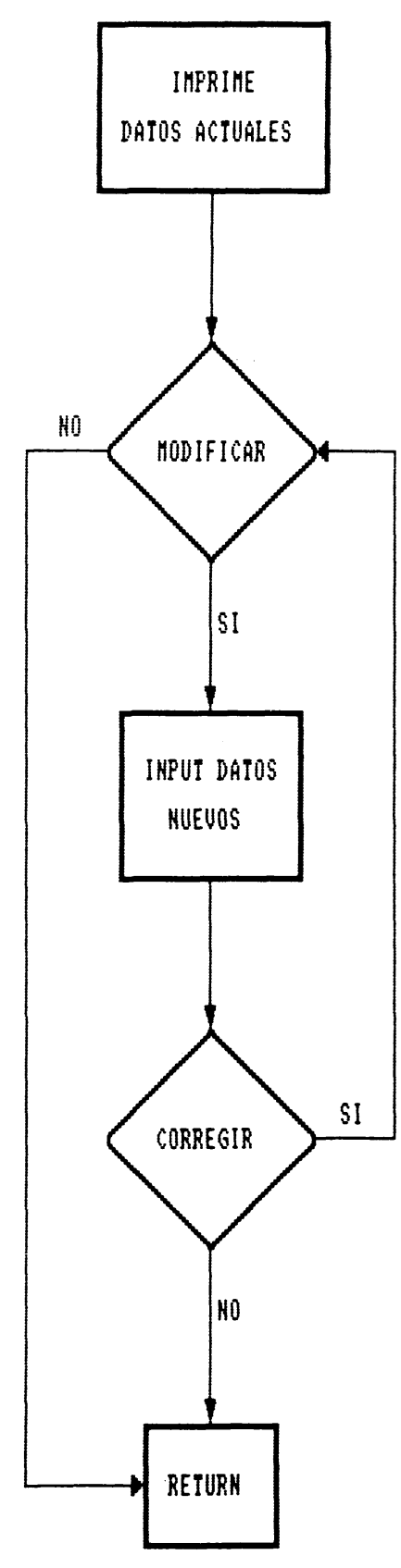









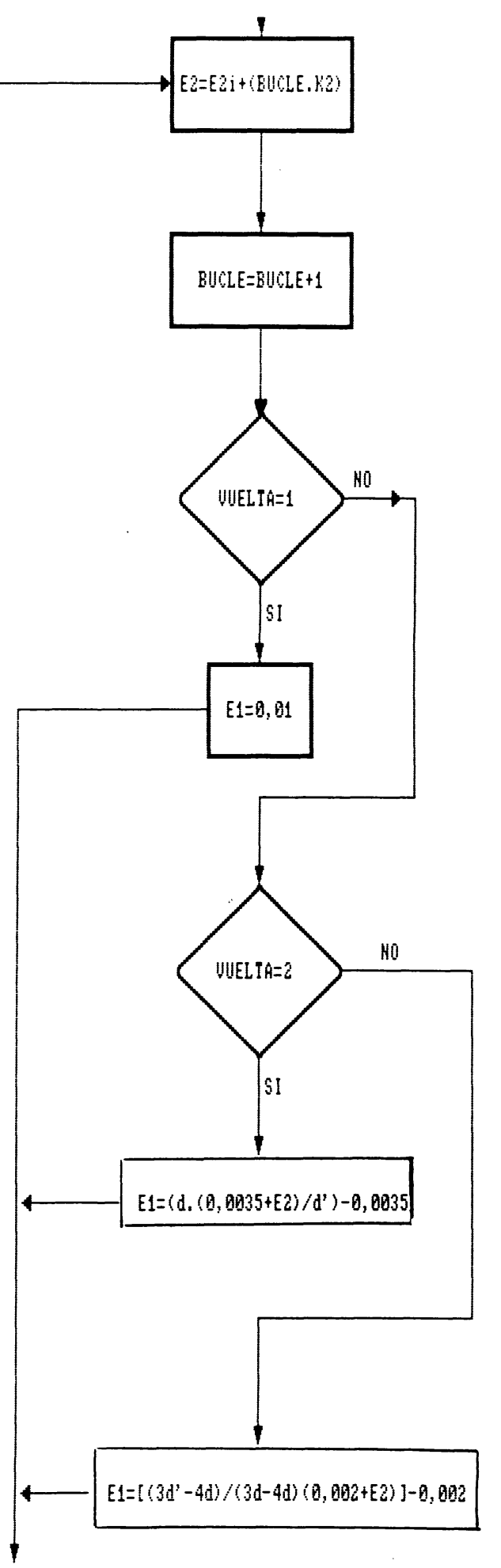

(c) Consejo Superior de Investigaciones Científicas Licencia Creative Commons 3.0 España (by-nc)

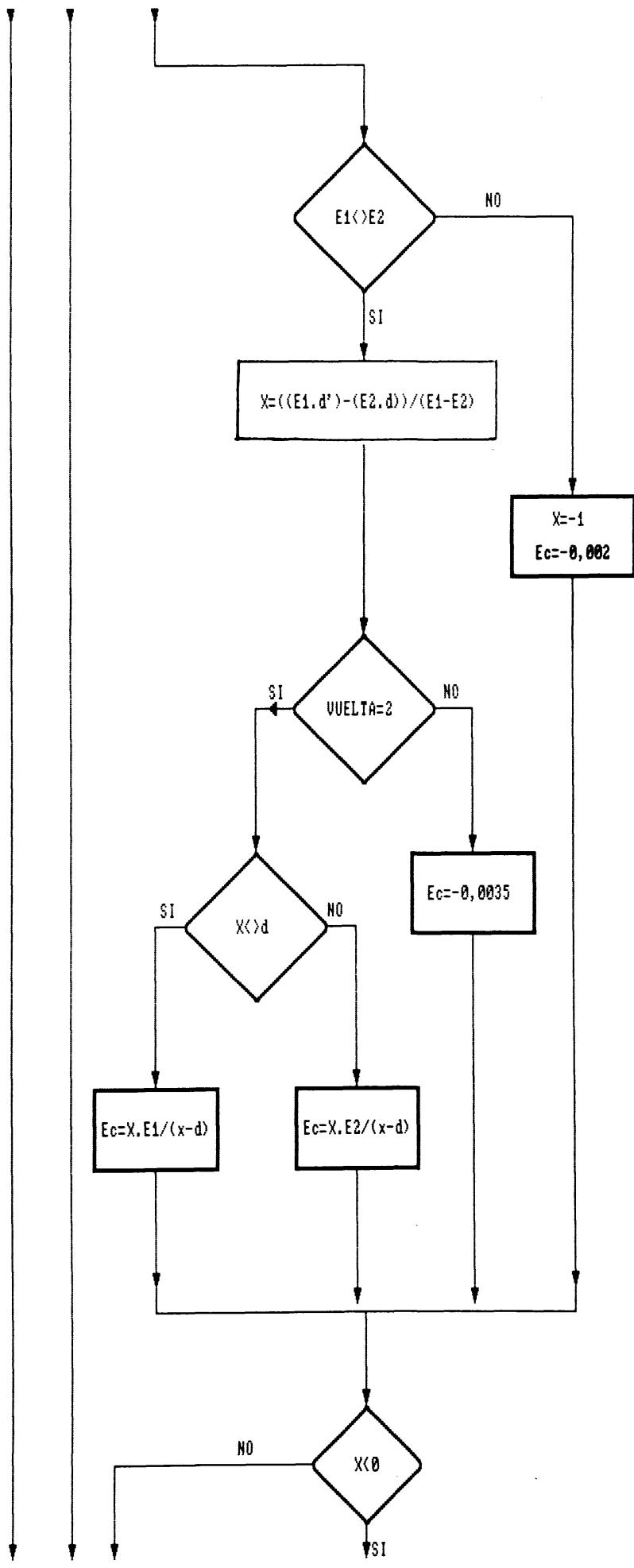






(C) Consejo Superior de Investigaciones Científicas Licencia Creative Commons 3.0 España (by-nc)

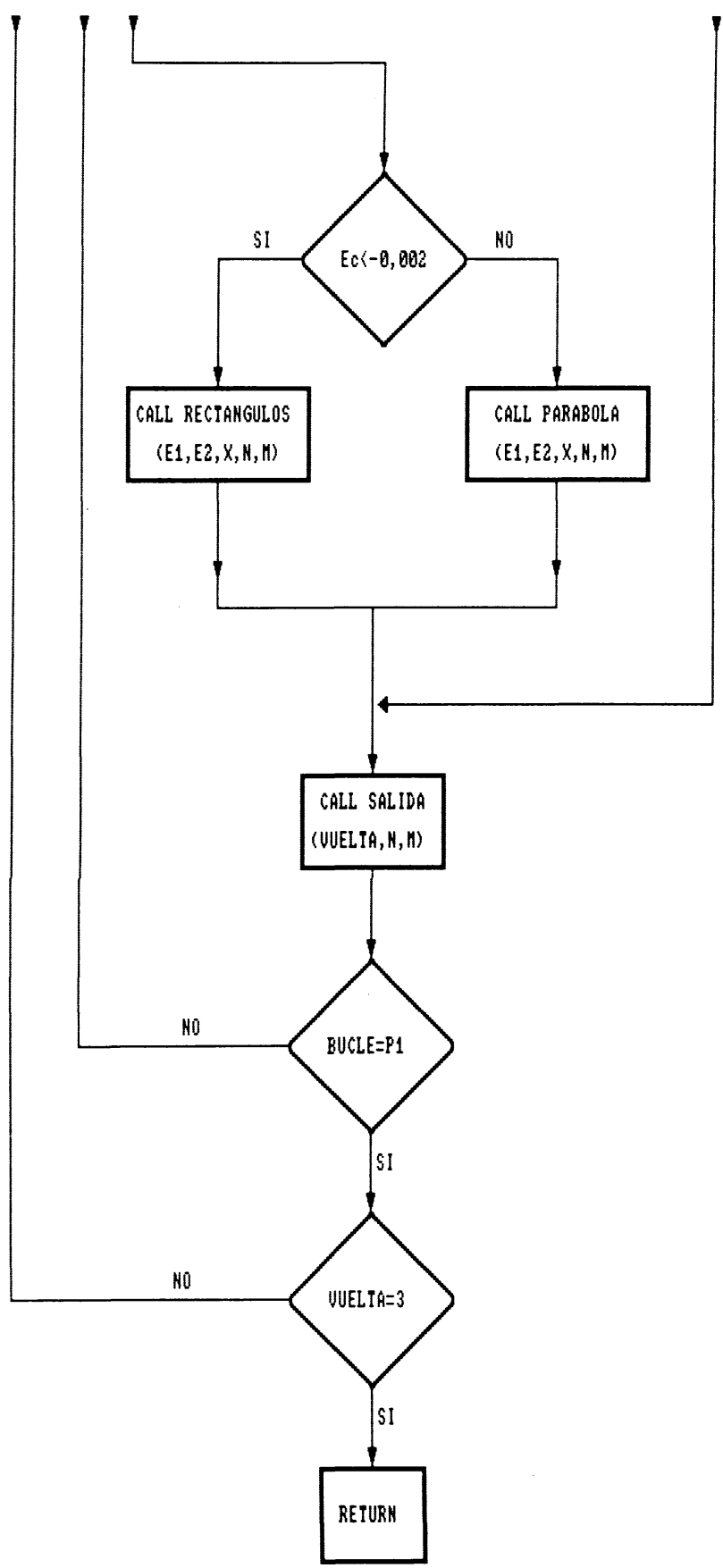

http://informesdelaconstruccion.revistas.csic.es 


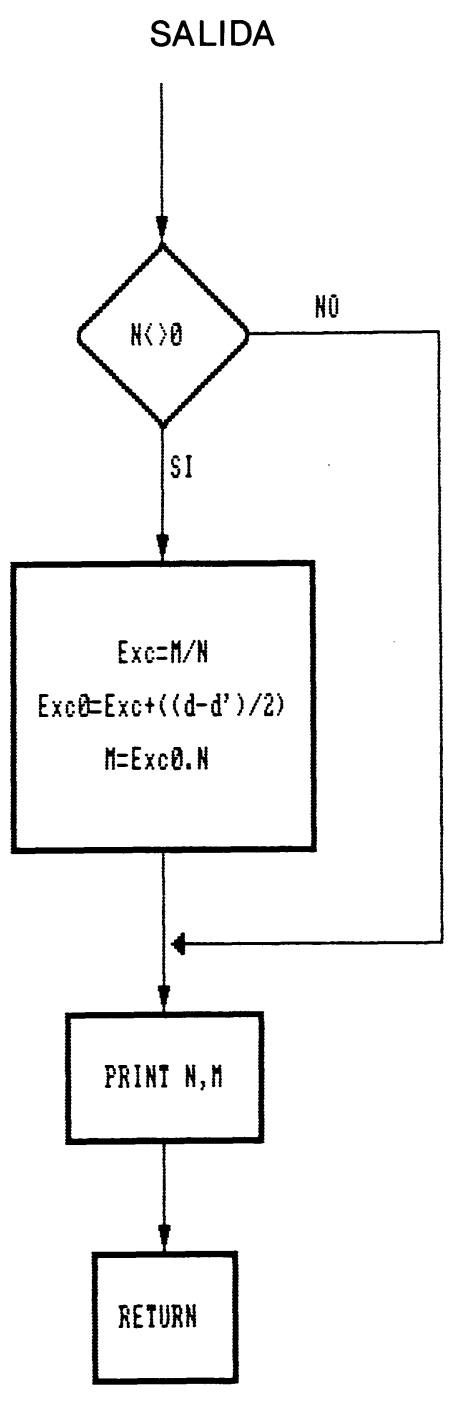

\section{Nota}

Debido a la extensión de este trabajo y a las características de INFORMES, éste se ha tenido que resumir y adaptarle al diseño de la revista.

Para mayor información los lectores pueden dirigirse a cualquiera de los autores al Dpto. de Construcción y Vías Rurales de la E.T.S. de Ingenieros Agrónomos de Madrid. 